


\begin{tabular}{|c|c|}
\hline$\Omega$ & U] \\
\hline Hannah Zhu', & $\begin{array}{l}\text { 'Dept of Paediatrics, King's College } \\
\text { Hospital NHS Foundation Trust, }\end{array}$ \\
\hline Preety Das ${ }^{2}$, & $\begin{array}{l}\text { London, UK } \\
{ }^{2} \text { Dept of Public He }\end{array}$ \\
\hline Ralph Woodhouse ${ }^{3}$, & $\begin{array}{l}\text { School of Public Health, Cambridge, } \\
\text { MA, USA }\end{array}$ \\
\hline Haytham Kubba ${ }^{4}$ & $\begin{array}{l}\text { 3Intensive Care Unit, National } \\
\text { Respiratory Centre, Danderyd } \\
\text { hospital, Danderyd, Sweden } \\
\text { 4Paediatric Otolaryngology, Royal } \\
\text { Hospital for Sick Children, } \\
\text { Clasgow, UK }\end{array}$ \\
\hline
\end{tabular}

$\equiv$

H. Zhu, King's College

London NHS Foundation

Trust, Paediatrics, Princess

Royal University Hospital,

Farnborough Common,

Orpington, London, BR6

8ND, UK hannah.h.zhu@gmail.com

\section{Improving the quality of tracheostomy care}

\section{Educational aims}

- To understand the current challenges in the care of tracheostomy patients

- To understand principles of quality improvement collaboration and how this can improve the quality of care for tracheostomy patients

\section{Summary}

The UK National Confidential Enquiry into Patient Outcomes and Death illustrates that there remains significant morbidity and mortality relating to patients with a tracheostomy, with much preventable harm. Challenges include the inherent complexity of the patient's underlying condition, wide variations in tracheostomy management, variable delivery of education for staff, patients and families, and difficult coordination of care between such a variety of individuals involved in performing, managing and ultimately removing tracheostomies.

Quality-improvement collaboratives are groups of institutions with a common purpose who work together to drive positive change. They help support clinicians in developing skills and teams necessary to design and sustain qualityimprovement cycles. They are a cost-effective way of rapidly disseminating improvement strategies and engaging in shared learning across institutions around the world. The Global Tracheostomy Collaborative aims to improve quality of care and outcomes through five interdependent key drivers: coordinated multidisciplinary team care, education, institution-wide protocols, family and patient-centred care, and metrics and outcomes using a specifically designed database.
Statement of Interest None declared.

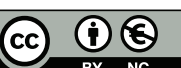

ERS 2014

HERMES syllabus links: E.1.6, F.6 


\section{Introduction}

Tracheotomies are increasingly performed in adults for upper airway obstruction, prolonged endotracheal intubation and airway protection or maintenance [1]. In children, a tracheotomy is less frequently performed than in adults, but prevalence is increasing due to increasingly complex conditions in children requiring a tracheostomy to facilitate long-term ventilation. Most paediatric tracheotomies are performed in the first year of life because of congenital anomalies of the airway, complications of intubation in premature infants or chronic neurological and respiratory disorders requiring prolonged or long-term mechanical ventilation $[2,3]$. In adults, the traditional surgical tracheotomy approach has been accompanied by percutaneous dilatational techniques in selected patients, especially in intensive care units (ICUs) [4]; however, this is not currently recommended in children [5].

Adult tracheotomies can be performed in the operating room or at the bedside in an ICU and may be done by various professionals including ear, nose and throat (ENT) surgeons, maxillofacial surgeons, general surgeons and intensivists. Most tracheotomies performed in adults in an ICU are temporary and may be inserted and removed during the same hospital stay. However, the vast majority of paediatric tracheotomies are performed by ENT or paediatric surgeons in the operating room with the child intubated under general anaesthesia. The tracheostomy is likely to remain in place for a period of months to years [6] and children with a tracheostomy experience lengthy and costly hospitalisations [7]

\section{Challenges in tracheostomy care}

The challenges in tracheostomy care are powerfully highlighted by the story from a daughter of a patient with a tracheostomy who died in hospital (box below) [8].

The UK National Confidential Enquiry into Patient Outcomes and Death (NCEPOD) investigates the full care pathway in patients with a new tracheostomy in hospital [9]. It illustrates that there remains significant morbidity and mortality associated with tracheostomy placement and management and makes key recommendations to improve care (table 1). Previous studies in the USA reported between 1 and $7 \%$ of patients experience an adverse event, and the majority of these occur after the first postoperative week. Many negative tracheostomyrelated outcomes are potentially preventable $[10,11]$. However, NCEPOD reported that $24 \%$ of patients in ICU and 31\% of ward patients experienced defined tracheostomy-related complications [9]. The UK National Patient Safety Agency identified 53 out of 1085 airway incidents related to tracheostomy incidents and 14 were classified as major or life threatening [12]. Indeed, the fourth UK National Audit Project showed that, in critical care, the most serious airway-related incidents related to tracheostomies [13].

The team performing the tracheotomy is frequently different to the team responsible for the ongoing management and removal of the tracheostomy, such as when a tracheotomy is performed by the ICU team and the patient is then discharged to the care of a general physician on a medical ward. The main challenges in adult tracheostomy care are related to ensuring safe and consistent decision making when there is such a variety

\section{Challenges in tracheostomy care: a relative's testimonial}

"So they called the ENT doctor but by the time he arrived she'd already died. They said that the tube had become blocked so she couldn't breathe and her oxygen levels had become really low and her heart had stopped. They did try to resuscitate her but she was too weak. That's when we found out the things that hadn't happened but should have happened for my mum. She should've had the trachy tube changed every few weeks but that didn't happen. The tube inside that should've been cleaned every few hours, that's supposed to help it stop getting blocked. She should've had special oxygen to help her breathing and I don't think she ever had that except when she was on intensive care. They did say that on the ward they hadn't had a patient with a trachy for about 2 years so it's no wonder they didn't know what they were doing." 
Table 1. Common complications of tracheostomies

\begin{tabular}{llc} 
Immediate (peri-operative) & Early $(<\mathbf{1}$ week) & Late ( $>\mathbf{1}$ week) \\
\hline Haemorrhage & Tube obstruction (mucus plug or blood) & Haemorrhage (local tissue trauma/ \\
Misplacement of tube & Partial or complete tube obstruction & Tracheal stenosis/ tracheomalacia/ \\
Tube occlusion & Infection & tracheocutaneous fistula \\
Loss of upper airway & Ulceration/necrosis of trachea, mucosal & Scar formation \\
Surgical emphysema & ulceration due to tube migration & Tube obstruction or accidental \\
Pneumothorax & Risk of occlusion in patients who have & decannulation \\
& difficulty extending their neck (obese or & Granulation tissue
\end{tabular}

of individuals involved in performing tracheotomies, and managing and ultimately removing tracheostomies. For example, what responsibility should an intensivist have if the tracheostomy she inserted percutaneously leads to complications on a medical ward three weeks after ICU discharge? Will she be informed that complications occurred? Should the ENT surgeon be responsible for managing this tracheostomy? Who decides which tracheostomy tubes are available in a particular hospital? The same challenges are present in paediatrics for inpatient care, but with the addition of the more frequent need for prolonged community care of these medically complex children.

Good management of a patient with a tracheostomy, both in the hospital and in the community, has a significant impact on quality of life [14]. An additional challenging area is the transition from inpatient to community care. Many patients have unnecessarily prolonged and costly inpatient stays due to lack of available home care [15]. Effective management involves effective coordination of care, education of the patient and the family, attentive care, training to recognise and manage tube obstruction, confidence in changing the tracheostomy tube, knowledge of the tube characteristics (i.e. size, settings and function), and adequate access to advice and support from the hospital team.

Wide variations in tracheostomy management exist between hospitals, as well as between hospital and community settings $[9,16,17]$. This results in a great deal of confusion amongst healthcare professionals on how to care for patients with a tracheostomy. Efforts to reduce variation in practice can improve clarity, facilitate training and should help to minimise complications, prevent some hospitalisations and reduce mortality. Many factors contributing to adverse events can be identified, analysed and addressed through systematic quality-improvement practices [10, 12].

\section{Barriers to wider implementation: lack of evidence and consensus}

There are many reasons why local improvements in tracheostomy care have not yet been widely implemented [18]. One is that tracheostomy care is shared between many healthcare teams from a variety of disciplines [4]. Few of these individuals will see tracheostomy-related complications on

NCEPOD key recommendations on improving tracheostomy care. Data from [9].

- Tracheostomy insertion should be recorded and coded as an operative procedure in all locations, and data collection should be as robust as in a theatre environment. This will facilitate planning and allow national review and audit.

- The diameter and length of the tracheostomy tube should be appropriate for the size and anatomy of the individual patient and should generally contain an inner tube.

- Training for bedside staff should include routine care as well as resuscitation procedures for tracheostomy patients. This should be supported by hospital wide guidance for tracheostomy care. Tube data as well as essential equipment should be clearly available at the bedside.

- Multidisciplinary care pathways which provide continuity of care between critical care and ward clinicians, and facilitate decannulation and discharge planning need to be established for all tracheostomy patients.

- Bedside staff caring for tracheostomy patients must be competent to recognise and manage common airway complications.

- Unplanned and night-time discharge of a patient with a tracheostomy is not recommended, particularly in patients with newly formed tracheostomy or those recently weaned from respiratory support. 
a regular basis unless they are a specialist in tracheostomy care (e.g. a tracheostomy specialist nurse). Thus, each individual or team has limited awareness of the potential problems in tracheostomy care, let alone the scale of those problems. Few institutions collect data on tracheostomy outcomes (such as mortality, length of stay, hospital readmission rate, tube blockage or accidental decannulation). If institutions are not aware of the problem, they will not have a sense of "ownership" or responsibility and will not realise the urgent need for improvement.

The evidence base is limited for many tracheostomy-related interventions because traditional research methodology (such as the randomised controlled trial) is rarely logistically or economically feasible [19]. There are very few validated quality and outcome measures specifically for tracheostomy care. Outcomes, such as length of stay, readmissions and mortality, are more influenced by the patients' underlying clinical condition (which is usually highly complex) than by the quality of their tracheostomy care [20]. Indeed, the majority of patients in the NCEPOD were ASA 3 or 4 (on the American Society of Anesthesiology patient classification status stratification) on admission [9]. As research evidence is lacking, it is difficult to justify the development of a clinical practice guideline, so we must rely on a consensus of expert opinions, such as the American Academy of Otolaryngology-Head and Neck Surgery (AAO-HNS) Clinical Consensus Statement on Tracheostomy Care [21]. However, local consensus is hard to achieve, as evidenced by the wide variation that exists in tracheostomy care both between and within institutions. As a result, local practice is often based on "common sense" and clinical experience.

\section{Quality-improvement collaboratives}

Despite these challenges, quality improvement collaboratives (QICs) can be highly effective in circumstances where the traditional multiple-site randomised clinical trial is impractical or cost prohibitive. QICs are groups of institutes that work together with a common purpose to drive positive change through data analysis and shared learning. They help support clinicians in developing skills and teams necessary to design and sustain quality-improvement cycles [22]. They are a cost-effective way of rapidly disseminating improvement strategies, and engaging in shared learning across institutions around the world. QICs have been shown to improve patient care in a variety of settings such as neonatal and paediatric intensive care units [23, 24].

\section{The Global Tracheostomy Collaborative: developing global alliance}

The Global Tracheostomy Collaborative (GTC) was formed in 2012 as the first quality improvement collaborative focused on tracheostomy care [25]. It is a non-profit organisation with a mission statement "to improve the quality of care and quality of life for every individual who has a temporary or long-term tracheostomy". It aims to improve tracheostomy care throughout a patient's journey, starting with inpatient care and extending to outpatient and community care (table 2) [26].

\section{Co-ordinated multidisciplinary team care}

The NCEPOD showed that there is currently much variation in clinical teams involved in tracheostomy care. Only $67.1 \%$ (318 out of 474) of ward patients with a tracheostomy were discussed at an MDT meeting. The composition of the inpatient MDT usually included physiotherapists (88\%) and speech and language therapists (91\%). However this was not universal and neither was the involvement of critical care outreach (43\%), dieticians (59\%) and specialist nurses (77\%) [9].

The NCEPOD recommended that multidisciplinary care pathways which provide continuity between critical care unit staff and ward clinicians, and facilitate decannulation and discharge planning need to be established for all tracheostomy patients. Members of a dedicated tracheostomy care team coordinate and assess patient progress as they approach decannulation. Such teams have shown documented improvements in several single-institution studies worldwide [27]. The advantages of the team approach include concentration of training and experience, clear lines of responsibility and ownership of the problem.

For example, HetTiGe et al. [28] in London described a ward-based tracheostomy care 
Table 2. Strategies to improving patient care throughout care period

\begin{tabular}{ll}
\hline Strategies & Improved outcomes \\
\hline $\begin{array}{l}\text { Inpatient } \\
\text { Create dedicated teams }\end{array}$ & Reduce morbidity and mortality \\
Perform ward rounds & \\
Establish checklists & Reduce morbidity and mortality \\
\hline $\begin{array}{l}\text { Outpatient } \\
\text { Create dedicated clinics }\end{array}$ & Reduce readmissions \\
$\quad$ Establish care plan & Improve outcomes in both inpatient and outpatient care \\
\hline $\begin{array}{l}\text { Collaborative and educational } \\
\text { Establish outcome measures }\end{array}$ & \\
Utilise a centralised database to measure outcomes & \\
Share data & \\
Use technological means to disseminate data and educate & \\
Data from [26]. &
\end{tabular}

bundle in a tertiary hospital, including implementation of a Tracheostomy Multidisciplinary Ward Team, which was associated with a reduction in serious tracheostomy-related critical events. These interventions reduced length of ICU and inpatient stay, whilst reducing time to tracheostomy tube decannulation [29,30]. In 2009, CAMERON et al. [31] in Australia introduced a tracheostomy review and management service (called "TRAMS"), which also demonstrated improved outcomes: patients spoke sooner, earlier decannulation and decreased length of acute stay with cost savings. PANDIAN et al. [32] in the USA showed that the introduction of a multidisciplinary percutaneous tracheostomy team, which identified, assessed and recruited patients to the multidisciplinary ward round prior to planned tracheostomy insertion, significantly reduced time between tracheostomy request and insertion, and decreased tracheostomy complications.

\section{Broad staff education}

The NCEPOD showed that, after tracheostomy insertion, patients are most commonly cared for on 2-4 different wards during their initial hospital stay, although 15 (7.2\%) out of 208 hospitals reported patients being moved to over 10 wards. In addition, 95 (58\%) out of 161 elective and 126 (65\%) out of 194 emergency tracheostomies were inserted at local district general hospitals, where staff have very limited experience with tracheostomy patients [9]. This highlights the huge challenge in ensuring that all bedside carers for tracheostomy patients are competent to recognise and manage common airway complications including tube obstruction or displacement.

The GTC aims to be a central repository, directing members to useful educational resources. For example, the UK National Tracheostomy Safety Project (NTSP) was founded in 2010 to improve the management of critical incidents involving tracheostomy and laryngectomy [12]. It has developed educational resources and emergency algorithms to improve management of tracheostomies, sourced directly from the NTSP [33]. These were developed by consensus, using existing guidelines, evidence and experiences of stakeholders and reinforced by open peer review. The final algorithms describe a universal approach to managing such emergencies and are designed to be followed by first responders [34].

In addition, the GTC aims to facilitate communication between teams caring for tracheostomy patients, disseminate highquality information and share best practice. The GTC also hosts online discussion groups and regular webinars around key tracheostomy quality issues. These allow practical application and sharing of expertise for continued learning and evidence-driven action.

\section{Institution-wide protocols}

A common challenge in clinical improvement is the delay between research discovery and clinical implementation [35]. The GTC aims to 


\section{Educational questions}

1. What are the three most common indications for tracheostomy placement in children?
a. Airway protection (secretions)
b. Congenital airway anomalies
c. Disorders requiring prolonged mechanical ventilation
d. Acute upper airway obstruction
e. Complications of intubation

2. Which two of the following are most commonly late ( $>_{1}$ week) complications following tracheostomy insertion?
a. Granulation tissue
b. Surgical emphysema
c. Pneumothorax
d. Misplacement of tube
e. Accidental decannulation

3. Which two of the following would benefit from forming a quality improvement collaborative compared with more traditional research methods like a randomised control trial?

a. Investigating the effect of a surgical checklist and post-operative care bundles on cardiac surgery processes and outcomes in developing countries.

b. Comparing outcomes of fluid resuscitation using crystalloid or colloid for sepsis in European countries.

c. Investigating the effect of implementing safety briefing and structured communication tools into inpatient handovers.

d. Investigating the effect of the human papillomavirus vaccine in preventing cervical cancer

e. Comparing the incidence and outcomes from breast cancer before and after introduction of a screening programme.

share information by rapidly disseminating evidence-based protocols from successful member hospitals, which other institutions may then adapt for their own use. For example, several institutions have developed local initiatives to improve standards of tracheostomy care. The literature has demonstrated the positive impact of qualityimprovement interventions that standardise clinical practice, such as evidence-based clinical care bundles and best practice guidelines [28, 36]. A few examples of NCEPOD recommendations are that consent forms and checklists should be used prior to tracheotomy wherever it is performed and that tracheostomy tube information as well as essential equipment should always be readily available at the bedside [9].

Regular communication enables member institutions to share interventions and discuss their personal successes and failures. Tracking of interventions and their effects over time using the GTC database will assist in developing and improving institution wide protocols.

\section{Family and patient-centred}

\section{care}

Patient and families are central to care in almost all healthcare settings, from primary care to ICUs $[37,38]$. Especially with the use of social media, their engagement is essential both in terms of partnership for their individual care but also quality improvement [39]. Patients and families often actively wish to engage in quality improvement and are some of the most inspiring champions and leaders, motivating healthcare professionals to change for the better [40]. Patient and family representation has been integral to the GTC from inception. The GTC offers tracheostomy patients and family members opportunities to connect with other patients and families, healthcare professionals, access patient-centred resources and share stories to increase awareness. Patients and families have informed the GTC that navigating the care system is often challenging, communication is often between the family and one provider rather than inter-disciplinary, and families are put in the middle of collaboration between specialists. To help facilitate this, they helped the GTC to formulate these five key drivers. In addition, they would like us to partner with them to formulate care plans, share all information and look to families to define "quality of life."

The GTC aims to improve quality of care and outcomes through five interdependent key drivers:

1. Co-ordinated multidisciplinary team care (MDT)

2. Broad staff education, sharing best practices

3. Institution wide protocols

4. Family and patient centered care

5. Metrics and outcomes

\section{Metrics and outcomes}

The GTC aims to support member institutions in facilitating meaningful and measurable clinical outcome changes. To do so, the GTC created a new database for collecting large amounts of tracheostomy data regarding adults and children worldwide over time in order to conduct robust statistical analysis. 
Member institutions enter data into the database, producing an invaluable resource for quality improvement and clinical research. The ability to analyse a longitudinal database of clinical outcomes for the first time in tracheostomy care will inform decisions about best practice and allow collective improvement in clinical outcomes.

In order to create this database, the GTC utilised Research Electronic Data Capture (REDCap) technology, a secure web-based data entry software, coupled with standardised GTC-specific data definitions [41]. This technology enables GTC member hospitals to enter retrospective clinical outcomes data for each tracheostomy insertion at their institute and share data with the collaborative in a safe and secure format. Institutions can opt for the full database (including dates) or a version that is devoid of any protected health information or potential patient identifiers. The preliminary GTC database will collect data on demographics (age, sex, chronic disease status) and key outcome variables (table 3) [26], which will be regularly reported to the group in anonymised form. The analysis allows for the heterogeneity of patients and uses the risk stratification of the Australian and New Zealand Intensive Care Society (ANZICS) as a basis for identifying comorbidities [42].

Initially, the GTC database will collect data exclusively on the key outcomes for the hospital admission during which the tracheostomy procedure occurred. In future, data will be collected for hospital readmissions, outpatient encounters, and ultimately for community-based
Table 3. Key clinical outcome measures of the Global Tracheostomy Collaborative

\section{Clinical outcome measure}

Indication for tracheostomy

Adverse events

Duration of stay in the intensive care unit

Duration of mechanical ventilation and tracheostomy tube

Total duration of hospital stay

Discharge disposition

Mortality rate

Data from [26].

care. The metrics will be regularly reviewed and quality process measures updated to reflect the most recent clinical protocols.

\section{Conclusion}

Few people would disagree that tracheostomy care could be improved and that many preventable complications and deaths occur. In a situation where robust research evidence is likely to be limited for the foreseeable future, the most efficient and effective route to change is through a quality improvement collaborative. The GTC has set itself an ambitious task: to bring patients, families and clinicians from many countries and many different backgrounds together to share of data and best practice to ultimately improve quality of care for all patients with a tracheostomy around the world.

\section{If you are interested in joining or finding out more about the GTC, please visit http://globaltrach.org}

\section{References}

1. Durbin Jr. CG. Tracheostomy: why, when, and how? Respir Care 2010; 55: 1056-1068.

2. Pereira KD, MacGregor AR, Mitchell RB. Complications of neonatal tracheostomy: a 5-year review. Otolaryngol Head and Neck Surg 2004; 131: 810-813.

3. Wallis C, Paton JY, Beaton S, et al. Children on longterm ventilatory support: 10 years of progress. Arch Dis Child 2011; 96: 998-1002.
4. Delaney A, Bagshaw SM, Nalos M. Percutaneous dilatational tracheostomy versus surgical tracheostomy in critically ill patients: a systematic review and meta-analysis. Crit Care 2006; 10: R55.

5. Durbin Jr. CG. Techniques for performing tracheostomy. Respir Care 2005; 50: 488-496.

6. Pérez-Ruiz E, Caro P, Pérez-Frías J, et al. Paediatric patients with a tracheostomy. A multicentre epidemiological study. Eur Respir J 2012; 40: 1502-1507.

\section{Suggested answers}
1. b, c, e.
2. a, e
3. a, c 
7. Zhu H, Das P, Roberson DW, et al. Hospitalizations in children with preexisting tracheostomy: A national perspective. Laryngoscope 2014 [In press DOI: 10.1002/lary.24797].

8. Global Tracheostomy Collaborative. Why do we need the GTC? www.globaltrach.org/about/why-do-weneed-the-gtc\#Improve. Date last updated: August 1,' 2014. Date last accessed: August 312014.

9. Wilkinson KA, Martin IC, Freeth $\mathrm{H}$, et al. On the right trach? A review of the care received by patients. UK National Confidential Enquiry into Patient Outcome and Death. Available from www.ncepod.org.uk/ 2014report1/downloads/On\%20the\%2oRight\%20Trach_. FullReport.pdf Date last updated: 2014. Date last accessed: October 17, 2014.

10. Das P, Zhu H, Shah RK, et al. Tracheotomy-related catastrophic events: results of a national survey. Laryngoscope 2012; 122: 30-37.

11. Halum SL, Ting JY, Plowman EK, et al. A multiinstitutional analysis of tracheotomy complications. Laryngoscope 2012; 122: 38-45.

12. McGrath BA, Thomas AN. Patient safety incidents associated with tracheostomies occurring in hospital wards: a review of reports to the UK National Patient Safety Agency. Postgrad Med J 2010; 86: 522-525.

13. Cook TM, Woodall N, Harper J, et al. Fourth National Audit Project. Major complications of airway management in the UK: results of the Fourth National Audit Project of the Royal College of Anaesthetists and the Difficult Airway Society. Part 2: intensive care and emergency departments. Br J Anaesth 2011; 106: $632-642$.

14. Griffiths J, Barber VS, Morgan L, et al. Systematic review and meta-analysis of studies of the timing of tracheostomy in adult patients undergoing artificial ventilation. BMJ 2005; 330: 1243

15. Edwards EA, O'Toole M, Wallis C. Sending children home on tracheostomy dependent ventilation: pitfalls and outcomes. Arch Dis Child 2004; 89: 251-255.

16. Shah RK, Lander L, Berry JG, et al. Tracheotomy outcomes and complications: a national perspective. Laryngoscope 2012; 122: 46-50.

17. Zhu H, Das P, Brereton J, et al. Surveillance and management practices in tracheotomy patients. Laryngoscope 2012; 122: 46-50.

18. Mace AD, Patel NN, Mainwaring F. Current standards of tracheostomy care in the UK. Otolaryngologist 2006; 1: 37-39.

19. Barbato A, Bottecchia L, Snijders D. Tracheostomy in children: an ancient procedure still under debate. Eur Respir J 2012; 40: 1322-1323.

20. Berry JG, Graham RJ, Roberson DW, et al. Patient characteristics associated with in-hospital mortality in children following tracheotomy. Arch Dis Child 2010; 95: 703-710.

21. Mitchell RB, Hussey HM, Setzen G, et al. Clinical consensus statement: tracheostomy care. Otolaryngol Head Neck Surg 2013; 148: 6-20.

22. Nadeem E, Olin SS, Hill LC, et al. Understanding the components of quality improvement collaboratives: a systematic literature review. Milbank $Q$ 2013; 91: 354-394.

23. Horbar JD, Rogowski J, Plsek PE, et al. Collaborative quality improvement for neonatal intensive care. NIC/Q Project Investigators of the Vermont Oxford Network. Pediatrics 2001; 107: 14-22.

24. Goldstein B, Nadel S. The promise and potential of continuous improvement in the pediatric intensive care unit: the evolving story from the United Kingdom paediatric intensive care audit network. J Pediatr 2013; 163: 935-937.

25. Felton M, Yuan J, Liu C, et al. The Trachea Collaborative. Inaugural meeting report. Glasgow, Scotland. The Global Tracheostomy Collaborative.
26. Enamandram S, Peltz A, Arora A, et al. Global Tracheostomy Collaborative: the future of quality improvement strategies. Curr Otorhinolaryngol Rep 2014; 2; 13-19.

27. Hettige R, Arora A, Oldfield WLG, et al. Recent developments to improve the standards of tracheostomy care. Br J Intens Care 2013; 77-80.

28. Hettige R, Arora A, Ifeacho S, et al. Improving tracheostomy management through design, implementation and prospective audit of a care bundle: how we do it. Clin Otolaryngol 2008; 33: 488-491.

29. Arora A, Hettige R, Ifeacho $S$, et al. Driving standards in tracheostomy care: a preliminary communication of the St Mary's ENT-led multidisciplinary team approach. Clin Otolaryngol 2008; 33: 596-599.

30. Cetto R, Arora A, Hettige R, et al. Improving tracheostomy care: a prospective study of the multidisciplinary approach. Clin Otolaryngol 2011; 36: $482-488$.

31. Cameron TS, McKinstry A, Burt SK, et al. Outcomes of patients with spinal cord injury before and after introduction of an interdisciplinary tracheostomy team. Crit Care Resusc 2009; 11: 14-19.

32. Pandian V, Miller CR, Mirski MA, et al. Multidisciplinary team approach in the management of tracheostomy patients. Otolaryngol Head neck Surg 2012; 147: 684-691.

33. National Tracheostomy Safety Project. Information resource for safer management of patients with tracheostomies and laryngectomies. www.tracheostomy. org.uk. Date last updated: July 29, 2014. Date last accessed: August 292014.

34. McGrath BA, Bates L, Atkinson D, et al. Multidisciplinary guidelines for the management of tracheostomy and laryngectomy airway emergencies. Anaesthesia 2012; 67: 1025-1041.

35. Lenfant $C$. Shattuck lecture-clinical research to clinical practice - lost in translation? $N$ Engl J Med 2003; 349: 868-874.

36. Boesch RP, Myers C, Garrett T, et al. Prevention of tracheostomy-related pressure ulcers in children. Pediatrics 2012; 129: e792-e797.

37. Parsons S, Winterbottom A, Cross $\mathrm{P}$, et al. The quality of patient engagement and involvement in primary care. An Inquiry into the Quality of Genera Practice in England, 2010. Available from www. kingsfund.org.uk/sites/files/kf/field/field_document/ patient-engagement-involvement-gp-inquiry-researchpaper-mar11.pdf Date last updated: March 2011. Date last accessed: Oct 172014.

38. Agency for Healthcare Research and Quality. Guide to Patient and Family Engagement in Hospital Quality and Safety. www.ahrq.gov/professionals/ systems/hospital/engagingfamilies Date last updated: June 1, 2013. Date last accessed: August 31, 2014.

39. Bornkessel A, Furberg R, Lefebvre RC. Social media: opportunities for quality improvement and lessons for providers-a networked model for patient-centered care through digital engagement. Curr Cardiol Repiatr 2014; 16: 504 .

40. Micalizzi DA, Bismark M. The heart of health care: parents' perspectives on patient safety. Pediatr Clinic N Am 2012; 59: 1233-1246.

41. Harris PA, Taylor R, Thielke R, et al. Research electronic data capture (REDCap) - A metadata-driven methodology and workflow process for providing translational research informatics support. J Biomed Inform 2009; 42: 377-381.

42. Australian and New Zealand Intensive Care Society (ANZICS) Centre for Outcome \& Resource Evaluation (CORE). Australasian Outcomes Research Tool for Intensive Care (AORTIC) Manual. Melbourne, ANZICS, 2013 